\title{
Prognostic significance of pathological response after neoadjuvant chemotherapy or chemoradiation for locally advanced cervical
}

\section{carcinoma}

\author{
Myrna Candelaria ${ }^{1}$, José Chanona-Vilchis ${ }^{2}$, Lucely Cetina ${ }^{1}$, Diana Flores- \\ Estrada $^{1}$, Carlos López-Graniel ${ }^{3}$, Aaron González-Enciso ${ }^{3}$, David Cantú ${ }^{3}$, \\ Adela Poitevin ${ }^{4}$, Lesbia Rivera ${ }^{4}$, Jose Hinojosa ${ }^{4}$, Jaime de la Garza ${ }^{1}$ and \\ Alfonso Dueñas-Gonzalez*5
}

\begin{abstract}
Address: ${ }^{1}$ Division of Clinical Research, Instituto Nacional de Cancerología, Mexico City, ${ }^{2}$ Pathology Department, Instituto Nacional de Cancerología, Mexico City, ${ }^{3}$ Gynecology-Oncology Department, Instituto Nacional de Cancerología, Mexico City, ${ }^{4}$ Division of Radiotherapy, Instituto Nacional de Cancerología, Mexico city and ${ }^{5}$ Unidad de Investigación Biomédica en Cáncer, Instituto de Investigaciones Biomédicas, Universidad Nacional Autónoma de México, Instituto Nacional de Cancerología, Mexico City

Email: Myrna Candelaria -myrnac@prodigy.net.mx; José Chanona-Vilchis - jchanonav@incan.edu.mx;

Lucely Cetina - micuentalucely@yahoo.com; Diana Flores-Estrada -dfe15@yahoo.com.mx; Carlos López-Graniel - lgraniel@data.net.mx; Aaron González-Enciso - agencisoonco@ hotmail.com; David Cantú - dcantu3@excite.com; Adela Poitevin - adepoite@prodigy.net.mx; Lesbia Rivera - mrubi2@yahoo.com; Jose Hinojosa - johingom@yahoo.com; Jaime de la Garza - jgdelagarza@prodigy.net.mx; Alfonso DueñasGonzalez* - alfonso_duenasg@yahoo.com

* Corresponding author
\end{abstract}

\section{Published: 03 February 2006}

International Seminars in Surgical Oncology 2006, 3:3 doi:10.1 186/1477-7800-3-3
Received: 07 November 2005

Accepted: 03 February 2006

This article is available from: http://www.issoonline.com/content/3/l/3

(c) 2006 Candelaria et al; licensee BioMed Central Ltd.

This is an Open Access article distributed under the terms of the Creative Commons Attribution License (http://creativecommons.org/licenses/by/2.0), which permits unrestricted use, distribution, and reproduction in any medium, provided the original work is properly cited.

\begin{abstract}
Background: Cisplatin-based chemoradiation is the standard of care for locally advanced cervical cancer patients; however, neoadjuvant modalities are currently being tested. Neoadjuvant studies in several tumor types have underscored the prognostic significance of pathological response for survival; however there is a paucity of studies in cervical cancer investigating this issue.

Methods: Four cohorts of patients with locally advanced cervical carcinoma (stages IB2-IIIB); included prospectively in phase II protocols of either neoadjuvant chemotherapy with I) cisplatin-gemcitabine, 2) oxaliplatin-gemcitabine, 3) carboplatin-paclitaxel or 4) chemoradiation with cisplatin or cisplatin-gemcitabine followed by radical hysterectomy were analyzed for pathological response and survival.
\end{abstract}

Results: One-hundred and fifty three (86\%) of the 178 patients treated within these trials, underwent radical hysterectomy and were analyzed. Overall, the mean age was 44.7 and almost two-thirds were FIGO stage IIB. Pathological response rates were as follows: Complete (PCR) in 60 cases (39.2\%), Near-complete (p-Near-CR) in 24 (15.6\%) and partial (PPR) in 69 cases (45. I\%). A higher proportion rate of $\mathrm{PCR}$ was observed in patients treated with chemoradiotherapy (with cisplatin [19/40, 47.5\%]; or with cisplatin-gemcitabine [24/4I, 58.5\%] compared with patients receiving only chemotherapy, 6/23 (26\%), 3/8 (37.5\%) and 8/ $4 \mathrm{I}(19.5 \%)$ for cisplatin-gemcitabine, oxaliplatin-gemcitabine and carboplatin-paclitaxel respectively [p $=0.000 \mathrm{I}])$. A total of 29 relapses (18.9\%) were documented. The pathological response was the only factor influencing on relapse, since only $4 / 60(6.6 \%)$ patients with $p C R$ relapsed, compared with 25/93 (26.8\%) patients with viable tumor, either $p$ Near-CR or $p P R(p=0.001)$. Overall survival was $98.3 \%$ in patients with $P C R$ versus $83 \%$ for patients with either $p N e a r-C R$ or $P P R(p=0.009)$.

Conclusion: Complete pathological response but no Near-complete and partial responses is associated with longer survival in cervical cancer patients treated with neoadjuvant chemotherapy or chemoradiotherapy. 


\section{Background}

Cervical cancer remains as one of the biggest killers in women around the world. The epidemiology of cervical cancer is strongly related to the standard of living of populations, thus, underdeveloped countries present the higher mortality rates which can be as high as >70 per 100 000 inhabitants and most cases are diagnosed in locally advanced disease -stages IB2-IVA- according to the FIGO classification [1].

Five randomized studies have demonstrated that survival with radiation therapy alone is lower than with radiation therapy with concomitant chemotherapy based on cisplatin [2-6]. Afterwards, a meta-analysis corroborated these findings confirming that chemoradiation offers an absolute survival benefit at 5 years of $12 \%$ [7]. Thus, cisplatinbased chemoradiation was largely accepted as the standard of care for cervical cancer patients whose treatment requires radiation. However, not only concomitant chemoradiotherapy has shown benefit in locally advanced cervical cancer; a meta-analysis of neoadjuvant chemotherapy followed by radical hysterectomy has also shown an absolute benefit of $15 \%$ at 5-year survival [8]; currently, the EORTC is conducting a randomized phase III trial comparing these two treatment modalities. On the other hand, multimodal treatments incorporating radiation, chemotherapy and surgery must be investigated in the aim to further improve the prognosis [9].

Studies in breast carcinoma, one of the tumors most frequently treated with neoadjuvant chemotherapy have underscored the importance of achieving a major or complete pathological response to prolong survival. In general, higher pathological complete response rate correlates with better survival $[10,11]$. On these bases it can be hypothesized that more effective neoadjuvant modalities would produce longer survival by increasing the pathological complete response rates.

There exists limited information on the value of pathological response for predicting survival in cervical cancer patients treated with neoadjuvant modalities. In particular, the meaning of pathological complete, near-complete (microscopic residual) or partial response remains unsettled. To investigate this issue, we have reviewed the outcome of the patients that underwent surgery in our series of phase II studies of neoadjuvant chemotherapy or chemoradiation followed by radical hysterectomy.

\section{Methods}

Four phase II studies were performed on FIGO staged IB2IIIB patients diagnosed with cervical squamous cell carcinoma, adenocarcinoma or adenosquamous carcinoma at the National Institute of Cancerology. Patients had to meet the following criteria in order to be included: 1) No previous oncological treatment; 2 ) older than 18 years old and younger than 70 years old; 3 ) functional status of 0-2 according to the WHO classification; 4) normal hematological, hepatic, renal and respiratory function according to the following parameters: hemoglobin $>9 \mathrm{gr} / \mathrm{L}$, leukocyte count $>4000 / \mathrm{mm}^{3}$, and platelets $>100000 / \mathrm{mm}^{3}$; total bilirubin and transaminases $<1.5$ times the high normal value; normal serum creatinine; 5) normal posteroanterior radiograph of the thorax; and 6) informed consent. The Institutional Regulatory Boards of the National Institute of Cancerology approved the protocols. Patients with history of cancer, severe systemic or uncontrolled metabolic diseases, neuropathy or psychiatric disorders were excluded.

Clinical staging was performed without anesthesia at least by two physicians of the Gynecology and Radiotherapy Departments. In the case of disagreement, the evaluation of an additional examiner was asked.

\section{Treatment protocols}

Neoadjuvant cisplatin gemcitabine followed by radical hysterectomy Treatment, toxicity and response evaluation details have been previously published [12]. Patients received 3 courses every 21 days of cisplatin at $100 \mathrm{mg} / \mathrm{m}^{2}$ on day 1 , and $1000 \mathrm{mg} / \mathrm{m}^{2}$ of gemcitabine on days 1 and 8 .

\section{Neoadjuvant oxaliplatin gemcitabine followed by radical hysterectomy}

Treatment, toxicity and response evaluation details have been previously published [13]. Patients received 3 courses every 21 days of oxaliplatin at $130 \mathrm{mg} / \mathrm{m}^{2}$ on day 1 , and $1250 \mathrm{mg} / \mathrm{m}^{2}$ gemcitabine on days 1 and 8 .

Neoadjuvant carboplatin paclitaxel followed by radical hysterectomy Treatment, toxicity and response evaluation details have been previously published [14]. Patients received 3 courses every 21 days with carboplatin at a dose calculated according with an area under the curve (AUC) of 6, and paclitaxel at $175 \mathrm{mg} / \mathrm{m}^{2}$ both on day 1 .

Neoadjuvant chemoradiation with cisplatin or cisplatin gemcitabine followed by radical hysterectomy

Treatment, toxicity and response evaluation details have been previously published [15]. This was a randomized phase II study where we compared cisplatin (weekly at 40 $\mathrm{mg} / \mathrm{m} 2$ for six) versus cisplatin gemcitabine (weekly at 40 $\mathrm{mg} / \mathrm{m} 2$ and $125 \mathrm{mg} / \mathrm{m} 2$ respectively, both for six) concurrent to external beam radiation (50-56 Gy in 5 weeks with 2 Gy fractions) followed by radical hysterectomy.

In all these four trials, radical hysterectomy was performed within 5 weeks after the neoadjuvant treatment, if it was clinically considered that resection would be possible obtaining free surgical margins. In case of unresectable 
Table I: Clinical stage according to neoadjuvant protocol for operated patients

\begin{tabular}{|c|c|c|c|c|c|c|}
\hline \multirow[t]{2}{*}{ Neoadjuvant protocol } & \multicolumn{5}{|c|}{ Clinical stage } & \multirow[t]{2}{*}{ Total } \\
\hline & IB2 & IIA & IIB & IIIA & IIIB & \\
\hline cisplatin + gemcitabine & 2 & 3 & 14 & 0 & 4 & 23 \\
\hline carboplatin + paclitaxel & 7 & 7 & 22 & 1 & 4 & 41 \\
\hline oxaliplatin + gemcitabine & 2 & I & 4 & 0 & I & 8 \\
\hline CT/RT with cisplatin & 9 & 4 & 27 & 0 & 0 & 40 \\
\hline $\mathrm{CT} / \mathrm{RT}$ with cisplatin + gemcitabine & 8 & 9 & 24 & 0 & 0 & 41 \\
\hline Total & 28 & 24 & 91 & 1 & 9 & 153 \\
\hline
\end{tabular}

tumors, patients in trials of neoadjuvant chemotherapy received definitive treatment with radiation therapy (teletherapy and brachytherapy) plus $40 \mathrm{mg} / \mathrm{m}^{2}$ weekly cisplatin for 6 applications during teletherapy. In the neoadjuvant chemoradiation trial, patients with any pathological response less than complete or near-complete received brachytherapy. In addition, in the neoadjuvant cisplatin gemcitabine no postoperative radiation was used regardless of the pathological response, whereas in the oxaliplatin gemcitabine adjuvant chemoradiation was used in all cases with a pathological response less than complete; and in the carboplatin paclitaxel, only in those cases with partial response (no chemoradiation in complete or near-complete response).

\section{Follow-up}

Follow-up included pelvic examination every 3 months starting at treatment completion. Imaging studies, such as CT scan, were performed when considered clinically indicated.

\section{Evaluation of pathological response}

Pathological evaluation of tumor was done by a pathologist who was blinded to the treatment received by the patient. Complete cervix was included for analysis and the response was registered as follows: Patients with no residual viable tumor cells in the surgical specimen (primary tumor and lymph nodes TONOMO) were classified as having a pathological complete response ( $\mathrm{pCR})$; near-complete or microscopic response was defined with the presence of one or more foci of malignant viable cells measuring less than 1 millimeter and partial response when the residual tumor was larger than 1 millimeter.

\section{Statistical analysis}

Overall survival was estimated using the Kaplan-Meier method [16] from the date of diagnosis to date of death or last follow-up. The influence of variables on survival was analyzed using the Cox regression analysis [17]. All statistical tests were two-sided, with significance defined as $\mathrm{p}<$ 0.05. All analysis was performed using SPSS-10 software.

\section{Results \\ Characteristics of patients}

Out of 178 patients treated within these trials, 86\% (153 patients) were submitted to radical hysterectomy and are subjected to this analysis. Overall, the mean age was 44.78 (range 24-67) and almost two-thirds (91, 59.4\%) were IIB. Stage distribution according to protocol treatment is shown in table 1.

\section{Treatment and pathological response by group of treatment}

Neoadjuvant cisplatin gemcitabine followed by radical hysterectomy. This study included 41 patients or which 23 underwent surgery. Six 6 (26\%) patients had pCR and 17 pPR.

Neoadjuvant gemcitabine oxaliplatin followed by radical hysterectomy. This study included 10 patients. Only 8 of

Table 2: Distribution of pathological response in treatment groups

\begin{tabular}{|c|c|c|c|c|}
\hline Neoadjuvant protocol & Complete response & Near-complete & Partial response & Total \\
\hline cisplatin + gemcitabine & 6 & - & 17 & 23 \\
\hline carboplatin + paclitaxel & 8 & 4 & 29 & 41 \\
\hline oxaliplatin + gemcitabine & 3 & 3 & 2 & 8 \\
\hline CT/RT with cisplatin & 19 & 8 & 13 & 40 \\
\hline CT/RT with cisplatin + gemcitabine. & 24 & 9 & 8 & 41 \\
\hline Total & 60 & 24 & 69 & 153 \\
\hline
\end{tabular}


them underwent radical hysterectomy, of which three $(37.5 \%)$ patient attained pCR, three $(37.5 \%)$ had pNearCR and two pPR (25\%).

Neoadjuvant carboplatin paclitaxel followed by radical hysterectomy. This study included 43 patients. Of these patients, 41 (95\%) underwent radical hysterectomy obtaining pCR and pNear-CR in $8(19.5 \%)$ and $4(9.7 \%)$ patients, respectively. The remaining 29 (70.7\%) patients had pPR.

Preoperative chemoradiation with cisplatin or cisplatin gemcitabine. Eighty-three patients were included in this randomized trial. Of these 40 were in the cisplatin arm and all were operated; 43 received cisplatin and gemcitabine and 41 underwent surgery. Pathological complete, near-complete and partial responses were as follows: cisplatin arm: 19 (47.5\%), 8 (20\%) and 13 (32.5\%) whereas in the cisplatin gemcitabine arm rates were: 24 (58.5\%), 9 $(22.5 \%)$ and $8(19.5 \%)$ respectively.

Summarizing, pathological response rates were as follows: pCR in 60 cases (39.2\%), pNear-CR in 24 (15.6\%) and $\mathrm{pPR}$ in 69 cases $(49.2 \%)$. A higher proportion rate of pCR was observed in patients treated with chemoradiotherapy (with cisplatin [19/40, 47.5\%]; or with cisplatingemcitabine [24/41,58.5\%]) compared with patients receiving only chemotherapy, $6 / 23$ (26\%), 3/8 (37.5\%) and $8 / 41(19.5 \%)$ for cisplatin-gemcitabine, oxaliplatingemcitabine and carboplatin-paclitaxel respectively $(\mathrm{p}=$ $0.0001)$, table 2 .

\section{Relapse rate}

A total of 29 relapses (18.9\%) were documented. The analysis of relapse rate, according with the neoadjuvant treatment, and clinical stage showed no statistical significant difference among all modalities administered $(\mathrm{p}=$ $0.06)$, as well as initial clinical stage ( $\mathrm{p}=0.53)$. The pathological response was the only factor influencing on relapse, since only $4 / 60(6.6 \%)$ patients with pCR relapsed, compared with 5/24 (20.8\%) or 20/69 (28.9\%) in patients with $\mathrm{pNear}-\mathrm{CR}$, or $\mathrm{pPR}$, respectively $(\mathrm{p}=$ $0.001)$.

\section{Overall survival}

Survival analysis was restricted to the 153 patients that underwent radical hysterectomy. At a median follow-up time of 29.6 months (2.9-66.4) for all patients, 5-yearoverall survival was $98.3 \%$ in patients with pCR versus $83 \%$ for patients with either pNear-CR or pPR ( $p=0.009)$ (Figure 1a). Among the ones with no pCR no difference in 5 -year overall survival was found between patients with pNear-CR compared with those with pPR (Figure 1b). Cox multivariate analysis was done to underscore factors influencing on survival. The only prognostic factor influencing on overall survival was pathological response $(\mathrm{p}=0.008$, $95 \%$ CI: $1.103-1.953)$. Neither the type of neoadjuvant treatment ( $\mathrm{p}=0.639,95 \% \mathrm{CI}: 0.710-1.748)$, nor the initial clinical stage ( $\mathrm{p}=0.531,95 \%$ CI: 0.707-1.959): were factors determining the overall survival.

\section{Discussion}

Currently, cisplatin-based chemoradiation is the standard of care for locally advanced cervical carcinoma [9]. This combined treatment has of proven efficacy even in patients treated outside of clinical trials which is certainly important as this neoplasm mainly affects socially disadvantaged women who may not comply with the treatment [18]. Nevertheless, the 5-year survival of locally advanced cervical cancer patients is around $70 \%$ hence other therapeutic approaches must be tested in order to further improve prognosis. Among these, neoadjuvant chemotherapy and neoadjuvant chemoradiation are being tested in phase III randomized trials.

A number of studies of neoadjuvant chemotherapy or concurrent chemoradiation in several tumor types underscore the importance of achieving a major or complete pathological response to prolong survival. Although it has been argued that patients with no residual carcinoma have a better outcome because of selection bias rather than the effects of preoperative therapy, if a high pathological complete response rate correlates with better survival then it can be hypothesized that more effective neoadjuvant modalities would produce longer survival by increasing the pathological complete response rates.

There are some indications that at least for cervical cancer this may hold true. Chang et al., [19] performed a randomized trial in IB bulky patients of neoadjuvant chemotherapy using the "quick scheme" of cisplatin $50 \mathrm{mg} / \mathrm{m}^{2}$ day 1 , bleomycin $25 \mathrm{mg} / \mathrm{m}^{2}$ days $1-3$, and vincristine 1 $\mathrm{mg} / \mathrm{m}^{2}$ day 1 , repeated every 10 days for three courses against pelvic radiotherapy alone. They found no differences in survival which can be the result of the low rate $(4.6 \%)$ of pathological complete response rate. Other phase II trials using these "old regimens" report similar low rates of pathological responses; such as $6.6 \%$ by Singh et al., [20], 9.5\% by Porzio [21], 7\% by Kim [22], and 12\% by Benedetti-Panici et al., [23]. On the contrary, with newer regimens of chemotherapy incorporating drugs such as gemcitabine $[12,13]$, vinorelbine [24], paclitaxel $[14,25]$ or irinotecan [26], complete pathological response rates are higher which may lead potentially to better survival rates. In this sense, we have reported in a comparison of two consecutive phase II trials similar survival of neoadjuvant chemotherapy with cisplatin gemcitabine followed by surgery in which complete pathological response was $26 \%$ versus chemoradiation with cisplatin in locally advanced cervical carcinomas [27], but better 
survival with that neoadjuvant treatment than radiation alone [28]. Pending confirmation from the randomized phase III trial that is ongoing in the EORTC it is very likely that neoadjuvant chemotherapy followed by surgery at best would be equivalent that the current standard of chemoradiation. These data strongly suggest that neoadjuvant chemotherapy trials need to be even more effective to show superiority over standard chemoradiation. However, so far there are no indications of such superiority with current drugs or combinations. For instance, a phase III trial of neoadjuvant chemotherapy in cervical cancer comparing a doublet -ifosfamide and cisplatin versus a triplet -ifosfamide, cisplatin and paclitaxel found no better results on survival by the incorporation of a third drug [29]. These results have led investigators to assay neoadju-

Figure 1a

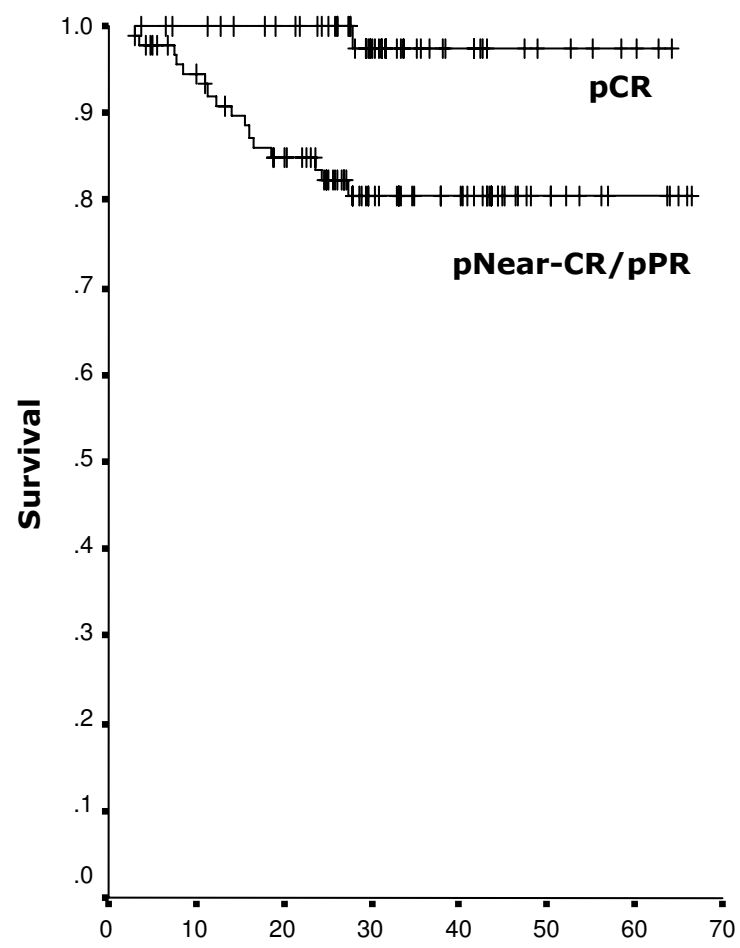

Months vant chemoradiation instead of only chemotherapy to improve pathological responses and survival.

Several prospective phase II trials of preoperative chemoradiation with cisplatin and 5-fluorouracil have been performed. Resbeut et al., reported a complete pathological response rate of $40 \%$ in 40 patients staged from IB to IVA [30]; Jurado et al., reported a complete pathological response rate of $67.5 \%$ in 40 patients [31] while the complete pathological response rate was $54.2 \%$ in 25 patients staged as IIB-IIIA in a third study [32]. Interestingly, the disease-free and overall survival these trials reported is very encouraging further supporting the hypothesis than prognosis can be improved by highly effective neoadjuvant therapies. In line with that, we performed a randomized phase II trial comparing cisplatin versus cisplatin

\section{Figure 1b}

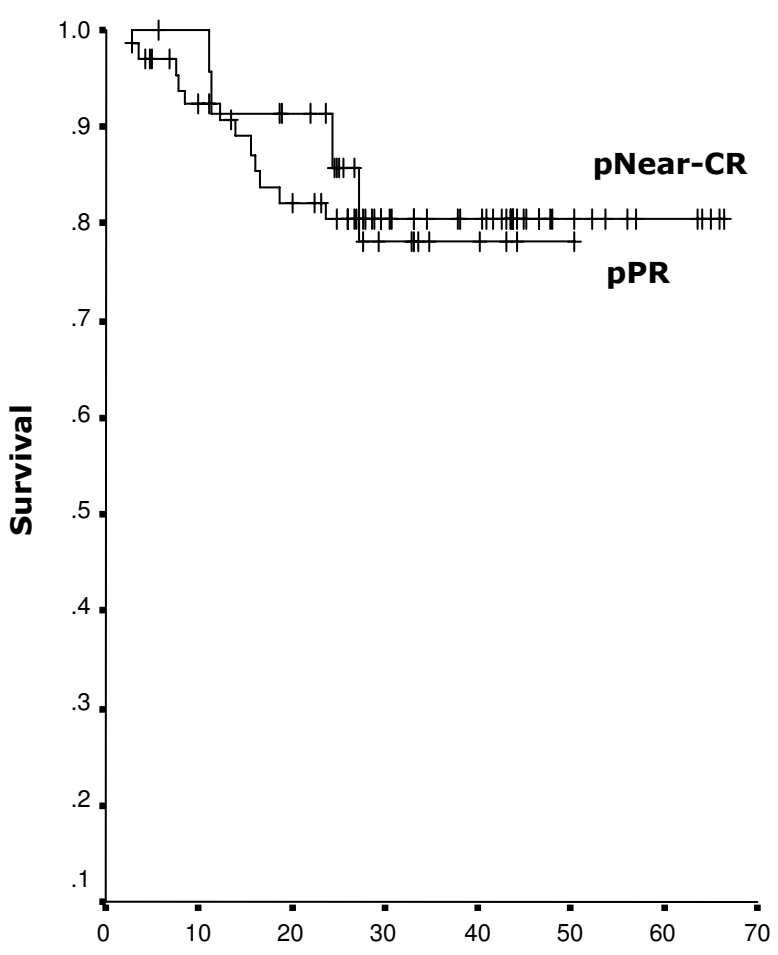

Months

\section{Figure I}

(A) Overall survival according to type of response. At a median follow-up time of 29.6 months (2.9-66.4), the survival for complete pathological response is $98.3 \%$ whereas is $83 \%$ for those with near-complete and partial response, $(p=0.009)$. (B) Overall survival for those with a response less than complete (near-complete and partial response). The survival for near-complete is $83.3 \%$ whereas is $82.3 \%$ for those with partial response $(p=0.820)$. 
and gemcitabine as sensitizers to radiation. Our results demonstrate that pathological complete responses can be indeed increased from $47.5 \%$ with cisplatin to $58.5 \%$ by adding gemcitabine, a powerful radiosensitizer to cisplatin [15].

There exists limited information on the value of pathological response for predicting survival in cervical cancer patients treated with neoadjuvant modalities. In particular, the meaning of pathological complete, near-complete (microscopic residual) or partial response remains unsettled. The results of this analysis shed some light on this issue. We found that for cervical cancer a complete pathological response by either neoadjuvant chemotherapy or neoadjuvant chemoradiation is indicative of very favorable survival whereas we found no different prognosis for those with no complete response irrespective of the "amount" of residual disease either microscopic (nearcomplete response) or minor or not change in disease (partial response). Resbeut et al., [30] made no distinction between complete and microscopic (near-complete) responses, instead they reported only complete or partial pathological response, nor integrated pathological response to their survival analysis, however; only one of the 16 patients with complete response had local recurrence suggesting its association with good prognosis. Jurado et al., defined complete pathological response as tumor eradication higher than $95 \%$ and found a 9-year local control rate of $100 \%$ versus $78 \%(\mathrm{p}=0.004)$ and overall survival of $93 \%$ versus $70 \%(p=0.038)$ for complete and partial responders respectively [31]. Finally, Mancuso's study registered complete, near-complete (as microscopic residual) and partial (as macroscopic residual) and reported no local failures among the 13 patients with complete response suggesting that complete responders have the most favorable prognosis [31,32]. The prognostic significance of pathological complete response is also suggested in the setting of pre-exenterative chemotherapy for recurrent cervical cancer patients. In such modality, only one of four patients with pathological complete response and two out of four with residual disease relapsed [33].

The discrepancy in the prognostic significance of complete versus near-complete also called as major response or microscopic residual is also observed in other tumors. In rectal and non-small cell lung treated with chemoradiation $[34,35]$ and bladder cancer treated with chemotherapy [36], complete and near-complete were predictive of better survival whereas in breast cancer the better prognosis is not observed for any response less than complete [37-39]. Whether the different prognostic meaning of pathological responses result from technical issues such as the number of patients and follow-up in studies, methods for analyzing and reporting the surgical specimens or result from the type of neoadjuvant treatment or even the type of tumor needs to be further studied. It is important however, to emphasize that among the studies on cervical cancer, the present report is the one reporting the higher number of patients and under two different neoadjuvant treatments which strengths our results on the prognostic significance of complete but no near-complete or partial pathological response.

\section{Conclusion}

Complete pathological response but no near-complete and partial responses are associated with very favorable survival in cervical cancer patients treated with neoadjuvant chemotherapy or chemoradiotherapy. These data strongly suggest that every effort must be made to obtain a high pathological complete response rate in order to improve survival. The value of adjuvant therapies for patients with a pathological response less than complete must be investigated in this subset of locally advanced cervical cancer patients treated under neoadjuvant protocols.

\section{Competing interests}

The author(s) declare that they have no competing interests.

\section{Authors' contributions}

MC conceived the study and analyzed the data and wrote the manuscript; J C-V did the pathological evaluation; LC was in charge of the chemotherapeutic management; AP, $\mathrm{LR}$ and $\mathrm{JH}$ performed the radiation management; DF-E participated in compilation of information and data management; CL-G, AG-E and DC participated in the surgical management of patients; $\mathrm{AD}-\mathrm{G}$ conceived the study and wrote the manuscript. All authors participated in the discussion and critically read the manuscript.

\section{Acknowledgements}

We thank to allied personnel that participated in the care of cervical cancer patients at our Institution.

\section{References}

I. Mohar A, Frias-Mendivil M: Epidemiology of cervical cancer. Cancer Invest 2000, 18:584-590.

2. Peters WA 3rd, Liu PY, Barrett R, Stock RJ, Monk BJ, Berek JS, Souhami L, Grigsby P, Gordon W Jr, Alberts DS: Concurrent chemotherapy and pelvic radiation therapy compared with pelvic radiation therapy alone as adjuvant therapy after radical surgery in high-risk early-stage cancer of the cervix. J Clin Oncol 2000, I8:1606-1613.

3. Morris M, Eifel PJ, Lu J, Grigsby PW, Levenback C, Stevens RE, Rotman M, Gerhenson DM, Mutch DG: Pelvic radiation with concurrent chemotherapy compared with pelvic and para-aortic radiation for high-risk cervical cancer. New Engl J Med 1999, 340: I I37-1 I43.

4. Keys HM, Bundy BN, Stehman FB, Muderspach LI, Chafe WE, Suggs $\mathrm{CL}$, Walker JL, Gersell D: A comparison of weekly cisplatin during radiation therapy versus irradiation alone each followed by adjuvant hysterectomy in bulky stage IB cervical carcinoma: a randomized trial of the Gynecology Oncology Group. New Engl J Med 1999, 340: I I54-I I6I. 
5. Rose PG, Bundy BN, Watkins EB, Thigpen JT, Deppe G, Maiman MA, Clarke-Pearson DL, Insalacos S: Concurrent cisplatin-based chemoradiation improves progression-free survival in advanced cervical cancer: results of a randomized Gynecologic Oncology Group study. New Engl J Med 1999 , 340:1144-1I53.

6. Whitney CW, Sause W, Bundy BN, Malfetano JH, Hannigan EV, Fowler WC Jr, Clarke-Pearson DL, Liao SY: A randomized comparison of fluorouracil plus cisplatin versus hydroxyurea as an adjunct to radiation therapy in stages IIB-IVA carcinoma of the cervix with negative para-aortic lymph nodes. A Gynecologic Oncology Group and Southwest Oncology Group Study. J Clin Oncol 1999, 17:1339-1348.

7. Green JA, Kirwan JM, Tierney JF, Symonds P, Fresco L, Collingwood $M$, Williams Cl: Survival and recurrence after concomitant chemotherapy and radiotherapy for cancer of the uterine cervix: A systematic review and meta-analysis. Lancet $200 \mathrm{I}$, 358:78I-786.

8. Neoadjuvant chemotherapy for cervical cancer meta-analysis collaboration: Neoadjuvant chemotherapy for locally advanced cervix cancer: a systematic review and meta-analysis of individual patient data from 21 randomized controlled trials. Eur J Cancer 2003, 39:2470-2486.

9. Duenas-Gonzalez A, Cetina L, Mariscal I, de la Garza J: Modern management of locally advanced cervical carcinoma. Cancer Treat Rev 2003, 29:389-399.

10. Stebbing JJ, Gaya A: The evidence-based use of induction chemotherapy in breast cancer. Breast Cancer 200I, 8:23-37.

II. Kurosumi M: Significance of histopathological evaluation in primary therapy for breast cancer - recent trends in primary modality with pathological complete response (PCR) as endpoint. Breast Cancer 2004, I I:139-47.

12. Dueñas-Gonzalez A, Lopez-Graniel C, González A, Reyes M, Mota A Muñoz D, Solorza G, Hinojosa LM, Guadarrama R, Florentino $R$, Mohar A, Melendez J, Maldonado V, Chanona J, Robles E, De la Garza J: A phase II study of gemcitabine and cisplatin combination as induction chemotherapy for untreated locally advanced cervical carcinoma. Ann Oncol 200 I, I 2:54 I-547.

13. Duenas-Gonzalez A, Lopez-Graniel C, Gonzalez A, Gomez E, Rivera L, Mohar A, Chanona G, Trejo-Becerril C, de la Garza J: Induction chemotherapy with gemcitabine and oxaliplatin for locally advanced cervical carcinoma. Am J Clin Oncol 2003, 26:22-25.

14. Duenas-Gonzalez A, Lopez-Graniel C, Gonzalez-Enciso A, Cetina L, Rivera L, Mariscal I, Montalvo G, Gómez E, De la Garza J, Chanona G, Mohar A: A phase II study of multimodality treatment for locally advanced cervical cancer: neoadjuvant carboplatin and paclitaxel followed by radical hysterectomy and adjuvant cisplatin chemoradiation. Ann Oncol 2003, I 4: I 278-84.

15. Duenas-Gonzalez A, Cetina-Perez L, Lopez-Graniel C, GonzalezEnciso A, Gomez-Gonzalez E, Rivera-Rubi L, Montalvo-Esquivel G Munoz-Gonzalez D, Robles-Flores J, Vazquez-Govea E, de La Garza J, Mohar A: Pathologic response and toxicity assessment of chemoradiotherapy with cisplatin versus cisplatin plus gemcitabine in cervical cancer: a randomized phase II study. Int J Radiat Oncol Biol Phys 2005, 6 I:817-823.

16. Kaplan EL, Meier P: Nonparametric estimation from incomplete observations. J Am Stat Assoc 1958, 53:457-48I.

17. Cox DR: Regression models and life-tables. J R Stat Soc $[B] 1972$, 34: $187-220$.

18. Cetina L, Rivera L, Hinojosa J, Poitevin A, Uribe J, Lopez-Graniel C, Cantu D, Candelaria M, de la Garza J, Duenas-Gonzalez A: The routine management of locally advanced cervical cancer with concurrent radiation and cisplatin, five-year results. $B M C$ Women's Health 2006, 6:3.

19. Chang TC, Lai CH, Hong JH, Hsueh S, Huang KG, Chou HH, Tseng CJ, Tsai CS, Chang JT, Lin CT, Chang HH, Chao PJ, Ng KK, Tang SG, Soong YK: Preliminary results of concurrent radiotherapy and chemotherapy with cis-platinum, vincristine and bleomycin in bulky, advanced cervical carcinoma. A pilot study. Gynecol oncol 1992, 44:182-188.

20. Singh KC, Agarwal A, Agarwal S, Rajaram S, Goel N, Agarwal N: "Quick Course" neoadjuvant chemotherapy with cisplatin, bleomycin and vincristine in advanced cervical cancer. Gynecol Obstet lnvest 2004, 58:109-113.
21. Porzio G, ficorella C, Toro G, Paris I, Recevuto E, Marchetti P: Shortterm weekly neoadjuvant chemotherapy in the treatment of locally advanced cervical cancer. Tumori 200I, 87:25-26.

22. Kim DS, Moon H, Hwang YY, Cho SH: Preoperative adjuvant chemotherapy in the treatment of cervical cancer stage Ib, Ila, and IIb with bulky tumor. Gynecol Oncol 1988, 29:32I-332.

23. Benedetti Panici P, Scambia G, Greggi S, Di Roberto P, Baiocchi G, Mancuso S: Neoadjuvant chemotherapy and radical surgery in locally advanced cervical carcinoma: a pilot study. Obstet Gynecol 1988, 7 I:344-348.

24. Lacava JA, Leone BA, Machiavelli M, Romero AO, Perez JE, Elem YL, Ferreyra R, Focaccia G, Suttora G, Salvadori MA, Cuevas MA, Acuna LR, Acuna JR, Langhi M, Amato S, Castaldi J, Arroyo A, Vallejo CT: Vinorelbine as neoadjuvant chemotherapy in advanced cervical carcinoma. J Clin Oncol 1997, I 5:604-609.

25. Zanetta G, Lissoni A, Pellegrino A, Sessa C, Colombo N, Gueli-Alletti $D$, Mangioni C: Neoadjuvant chemotherapy with cisplatin, ifosfamide and paclitaxel for locally advanced squamous-cell cervical cancer. Ann Oncol 1998, 9:977-980.

26. Sugiyama T, Nishida T, Kumagai S, Nishio S, Fujiyoshi K, Okura N Yakushiji M, Hiura M, Umesaki N: Combination therapy with irinotecan and cisplatin as neoadjuvant chemotherapy in locally advanced cervical cancer. Br J Cancer 1999, 81:95-98.

27. Duenas-Gonzalez A, Lopez-Graniel C, Gonzalez-Enciso A, Mohar A, Rivera L, Mota A, Guadarrama R, Chanona G, De la Garza J: Concomitant chemoradiation versus neoadjuvant chemotherapy in locally advanced cervical carcinoma. Results from two consecutive phase II trials. Ann Oncol 2002, 13:1212-1219.

28. Duenas-Gonzalez A, Lopez-Graniel CM, Mota A, Mohar A: Neoadjuvant chemotherapy followed by surgery in locally advanced cervical carcinoma. J Clin Oncol 2002, 20:2908-2909.

29. Buda A, Fossati R, Colombo N, Fei F, Floriani I, Gueli Alletti D, Katsaros D, Landoni F, Lissoni A, Malzoni C, Sartori E, Scollo P, Torri V, Zola P, Mangioni C: Randomized trial of neoadjuvant chemotherapy comparing paclitaxel, ifosfamide, and cisplatin with ifosfamide and cisplatin followed by radical surgery in patients with locally advanced squamous cell cervical carcinoma: the SNAPOI (Studio Neo-Adjuvante Portio) Italian Collaborative Study. J Clin Oncol 2005, 23:4l37-4I45.

30. Resbeut M, Cowen D, Viens P, Noirclerc m, Perez T, Gouvernet ], Delpero JR, Gamerre M, Boubli L, Houvenaeghel G: Concomitant chemoradiation prior to surgery in the treatment of advanced cervical carcinoma. Gynecol Oncol 1994, 54:68-75.

31. Jurado M, Martinez-Monge R, Garcia-Foncillas J, Azinovic I, Aristu J, Lopez-Garcia G, Brugarolas A: Pilot study of concurrent cisplatin, 5-fluorouracil, and external beam radiotherapy prior to radical surgery $+/$ - intraoperative electron beam radiotherapy in locally advanced cervical cancer. Gynecol Oncol 1999, 74:30-37.

32. Mancuso S, Smaniotto D, Benedetti-Panici P, Favale B, Greggi S, Manfredi R, Margariti PA, Morganti AG, Scambia G, Tortoreto F, Valentini V, Cellini N: Phase I-II trial of preoperative chemoradiation in locally advanced cervical carcinoma. Gynecol Oncol 2000, 78:324-328.

33. Lopez-Graniel C, Dolores R, Cetina L, Gonzalez A, Cantu D, Chanona J, Uribe J, Candelaria M, Brom R, de la Garza J, DuenasGonzalez A: Pre-exenterative chemotherapy, a novel therapeutic approach for patients with persistent or recurrent cervical cancer. BMC Cancer 2005, 5: I I8.

34. Ryan R, Gibbons D, Hyland JM, Treanor D, White A, Mulcahy HE, O'Donoghue DP, Moriarty M, Fennelly D, Sheahan K: Pathological response following long-course neoadjuvant chemoradiotherapy for locally advanced rectal cancer. Histopathology 2005 , 47: $|4|-\mid 46$

35. Trodella L, Granone P, Valente S, Margaritora S, Macis G, Cesario A, D'Angelillo RM, Valentini V, Corbo GM, Porziella V, Ramella S, Tonini G, Galetta D, Ciresa M, Vincenzi B, Cellini N: Neoadjuvant concurrent radiochemotherapy in locally advanced (IIIA-IIIB) nonsmall-cell lung cancer: long-term results according to downstaging. Ann Oncol 2004, I 5:389-398.

36. Splinter TA, Scher HI, Denis L, Bukowski R, Simon S, Klimberg I, Soloway $M$, Vogelzang $N$, van Tinteren $H$, Herr $H$ : The prognostic value of the pathological response to combination chemotherapy before cystectomy in patients with invasive bladder cancer. European Organization for Research on Treatment of Cancer - Genitourinary Group. J Urol 1992, 147:606-608. 
37. Chollet P, Amat S, Cure H, de Latour M, Le Bouedec G, Mouret-Reynier MA, Ferriere JP, Achard JL, Dauplat J, Penault-Llorca F: Prognostic significance of a complete pathological response after induction chemotherapy in operable breast cancer. $\mathrm{Br} J \mathrm{Can}$ cer 2002, 86:104I-1046.

38. Brain E, Garrino C, Misset JL, Carbonero IG, Itzhaki M, Cvitkovic E, Goldschmidt E, Burki F, Regensberg C, Pappo E, Hagipantelli R, Musset $M$ : Long-term prognostic and predictive factors in 107 stage II/III breast cancer patients treated with anthracyclinebased neoadjuvant chemotherapy. $\mathrm{Br} J$ Cancer 1997, 75:1360-1367.

39. Ring AE, Smith IE, Ashley S, Fulford LG, Lakhani SR: Oestrogen receptor status, pathological complete response and prognosis in patients receiving neoadjuvant chemotherapy for early breast cancer. Br J Cancer 2004, 9 I:20 I2-20I7.

Publish with Bio Med Central and every scientist can read your work free of charge

"BioMed Central will be the most significant development for disseminating the results of biomedical research in our lifetime. "

Sir Paul Nurse, Cancer Research UK

Your research papers will be:

- available free of charge to the entire biomedical community

- peer reviewed and published immediately upon acceptance

- cited in PubMed and archived on PubMed Central

- yours - you keep the copyright

Submit your manuscript here:

http://www.biomedcentral.com/info/publishing_adv.asp 